\title{
Tenomodulin knockout mice exhibit worse late healing outcomes with augmented trauma-induced heterotopic ossification of Achilles tendon
}

Manuel Delgado Caceres ${ }^{1}$, Katharina Angerpointner ${ }^{2}$, Michael Galler ${ }^{3}$, Dasheng Lin ${ }^{4}$, Philipp A. Michel ${ }^{5}$, Christoph Brochhausen ${ }^{6}$, Xin Lu ${ }^{7}$, Adithi R. Varadarajan ${ }^{7}$, Jens Warfsmann ${ }^{7}$, Richard Stange ${ }^{8}$, Volker Alt ${ }^{1,9}$, Christian G. Pfeifer ${ }^{1,9}$ and Denitsa Docheva (iD ${ }^{1,10 凶}$

(c) The Author(s) 2021

\begin{abstract}
Heterotopic ossification $(\mathrm{HO})$ represents a common problem after tendon injury with no effective treatment yet being developed. Tenomodulin (Tnmd), the best-known mature marker for tendon lineage cells, has important effects in tendon tissue aging and function. We have reported that loss of Tnmd leads to inferior early tendon repair characterized by fibrovascular scaring and therefore hypothesized that its lack will persistently cause deficient repair during later stages. Tnmd knockout (Tnmd $\left.{ }^{-/}\right)$and wildtype (WT) animals were subjected to complete Achilles tendon surgical transection followed by end-to-end suture. Lineage tracing revealed a reduction in tendon-lineage cells marked by ScleraxisGFP, but an increase in alpha smooth muscle actin myofibroblasts in $\mathrm{Tnmd}^{-1-}$ tendon scars. At the proliferative stage, more pro-inflammatory M1 macrophages and larger collagen II cartilaginous template were detected in this group. At the remodeling stage, histological scoring revealed lower repair quality in the injured $\mathrm{Tnmd}^{-1-}$ tendons, which was coupled with higher HO quantified by micro-CT. Tendon biomechanical properties were compromised in both groups upon injury, however we identified an abnormal stiffening of non-injured Tnmd ${ }^{-/-}$tendons, which possessed higher static and dynamic E-moduli. Pathologically thicker and abnormally shaped collagen fibrils were observed by TEM in $\mathrm{Tnmd}^{-/-}$tendons and this, together with augmented HO, resulted in diminished running capacity of Tnmd ${ }^{-/-}$mice. These novel findings demonstrate that Tnmd plays a protecting role against trauma-induced endochondral $\mathrm{HO}$ and can inspire the generation of novel therapeutics to accelerate repair.
\end{abstract}

Cell Death and Disease (2021)12:1049; https://doi.org/10.1038/s41419-021-04298-z

\section{INTRODUCTION}

Heterotopic ossification $(\mathrm{HO})$ is the process of ectopic cartilage formation followed by endochondral ossification of soft tissues such as tendons [1, 2]. Following open Achilles tendon reconstructions, up to $28 \%$ of patients suffer from $\mathrm{HO}[3,4]$. Trauma-induced HO significantly impacts tendon viscoelastic properties $[5,6]$, leading to serious decrease in patients quality of life. To date, no satisfactory therapeutic targets have been developed. The natural healing process of tendons, divided in early (inflammatory) and late (proliferative and remodeling) stages, is known to be inefficient, long-lasting and influenced by anatomic location and local mechanical environment [7]. Dysregulated inflammatory response in tendon lesions drives persistent fibrosis, misguided stem/progenitor cell differentiation and leads to $\mathrm{HO}[8,9]$. The discrete molecular mechanisms and origin, existence and functions of different cell types throughout the long repair process are still not fully understood. Tendon-resident cells are marked by the expression of Scleraxis $(S c x)$ [10], and their lineage tracking by using Scleraxis-GFP (ScxGFP) reporter mice [11] has been crucial for the identification of cells triggering intrinsic tissue repair [12-15]. On the other hand, extrinsic epitenon/ paratenon cells, expressing alpha smooth muscle actin (aSMA), and persistent pro- inflammatory M1 macrophages, have been shown to initiate pathologic scarring, chronic low-grade inflammation, poor tissue remodeling and $\mathrm{HO}[12,15,16]$.

Tenomodulin (Tnmd) gene, predominantly expressed in tendons and ligaments, encodes a type II transmembrane glycoprotein with a cleavable C-terminal cysteine-rich domain, that is subsequently deposited in the extracellular matrix (ECM) proximal to type I collagen fibrils [17-19]. The C-terminal hydrophobic tail

\footnotetext{
${ }^{1}$ Experimental Trauma Surgery, Department of Trauma Surgery, University Regensburg Medical Centre, Regensburg, Germany. ${ }^{2}$ Hand, Elbow and Plastic Surgery Department, Schön Klinik München Harlaching, Munich, Germany. ${ }^{3}$ Department of Trauma Surgery, Caritas Hospital St. Josef, Regensburg, Germany. ${ }^{4}$ Orthopaedic Center of People's Liberation Army, The Affiliated Southeast Hospital of Xiamen University, Zhangzhou, China. ${ }^{5}$ Department of Trauma-, Hand-, and Reconstructive Surgery, University Hospital Münster, Münster, Germany. ${ }^{6}$ Institute of Pathology, University of Regensburg, Regensburg, Germany. ${ }^{7}$ Division of Personalized Tumor Therapy, Fraunhofer Institute for Toxicology and Experimental Medicine, Regensburg, Germany. ${ }^{8}$ Department of Regenerative Musculoskeletal Medicine, Institute for Musculoskeletal Medicine, University Hospital Münster, Westfälische Wilhelms-University, Münster, Germany. ${ }^{9}$ Clinic and Policlinic for Trauma Surgery, University Regensburg Medical Centre, Regensburg, Germany.

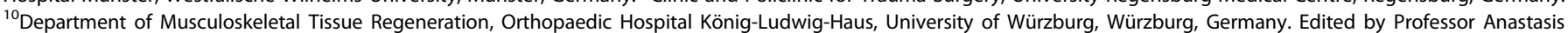
Stephanou. ${ }^{凶}$ email: denitsa.docheva@ukr.de

Edited by Professor Anastasis Stephanou.
}

Received: 8 July 2021 Revised: 5 October 2021 Accepted: 7 October 2021

Published online: 05 November 2021 
exerts a dual role stimulating proliferation of tendon cells [20], whilst inhibiting vascular cell migration [21]. It has also been demonstrated that Tnmd regulates the proliferation, senescence and differentiation of tendon stem/progenitor cells (TSPC) [22]. Moreover, we have previously shown that TSPCs derived from Tnmd-knockout mice exhibit defective in vitro collagen remodeling [23]. Further, a significant shift towards thicker and stiffer collagen fibrils was identified within non-injured mutant Achilles tendons $[20,24]$, resulting in profoundly hampered performance in forced endurance running tests [24]. Next, when subjected to surgically induced full thickness Achilles tendon rupture $[25,26]$, $\mathrm{Tnmd}^{-1-}$ mice displayed inferior scar tissue characterized by significantly reduced cell density and proliferation, increased vessel infiltration, and accumulation of M1 macrophages, adipocytes and erroneous ECM after 8 days [26]. Here, we implemented the established surgical model and carried out a longitudinal investigation up to day 100 with $\mathrm{Tnmd}^{-1-}$ and wild type (WT) animals, by combining lineage tracking, immuno-histomorphometry, transmission electron microscopy (TEM), micro-CT ( $\mu C T)$, viscoelastic biomechanical testing, voluntarily running recording, and single cell RNA-sequencing to diagnose for the first time transcriptional differences between both genotypes. Based on our previous findings we hypothesized that the lack of Tnmd will persistently cause deficient repair during the later proliferative and remodeling stages, and will lead to a significant endochondral heterotopic ossification of Achilles tendons.

\section{MATERIAL AND METHODS \\ Study approval}

All procedures regarding animal handling, husbandry, surgical model, pre-, and postoperative animal care were approved by the Animal Care and Use Committee of the Lower Franconia Government, Wuerzburg, Germany (Grant No. 55.2-2532-2-466). In the group size calculations, for the type one error and power, the standard values $\alpha=0.05$ and $1-\beta=0.8$ were used, respectively.

\section{Animal model and surgical procedure}

Constitutive Tnmd knockout (Tnmd ${ }^{-1-}$ ) mice and WT littermates, as well as SCXGFP mice were previously described by Docheva et al. [20] and Pryce et al. [11], respectively. Conditional Tnmd flox/flox strain is not yet available. Tnmd gene is located on the X-Chromosome, therefore the WT group comprised hemizygous male, homozygous-, and heterozygous female mice. All analyzed animals were maintained on the C57BL/6 J background. The experimental design and the group distribution including $\mathrm{n}$-numbers are shown in Supplementary Fig. $1 \mathrm{~A}$. Skeletally mature animals (6-month old) were operated according to Lin et al. [26]. Detailed description of the surgical procedure provided in Supplementary Information and Supplementary Fig. 1B, C.

\section{Immunohistochemistry and histomorphometry}

Mouse hindlimbs (exact n-numbers shown as dot plot as well as given in figure legends) were fixed overnight in fresh $4 \%$ paraformaldehyde (PFA, Merck, Darmstadt, Germany) in phosphate-buffered saline (PBS; pH 7.4) or in $95 \%$ ethanol- $5 \%$ glacial acetic acid. Specimens were decalcified in $10 \%$ ethylen diamine tetraacetic acid (EDTA)/phosphate buffered saline (PBS) pH 8.0 (Sigma-Aldrich, Munich, Germany) for 4 weeks, embedded in cryoprotective media and sectioned at $10 \mu \mathrm{m}$, every $10^{\text {th }}$ slide was stained with Hematoxylin-Eosin following standard protocol. Tissue sections with equivalent regional planes between genotypes were selected for detailed investigation. Histological scoring was carried out by two independent observers at day $21 \quad(n=10 /$ genotype $)$ and $100 \quad(n=11 /$ genotype $)$ according to Stoll et al. [27] (Supplementary Table 1). Detailed description of immunohistochemistry, immunofluorescence stainings and automated quantitative image analysis provided in Supplementary information.

\section{Transmission electron microscopy (TEM)}

Injured and contralateral Achilles tendons were explanted at day 21 and 100 post-injury ( $n=3$ genotype/time point), fixed in Karnovsky ( 0.1 M cacodylatebuffer with $2.5 \%$ glutaraldehyde and $2 \%$ paraformaldehyde). Ultrathin sections $(0.08 \mu \mathrm{m})$ were analyzed in a LEO912AB transmission electron microscope (Zeiss, Oberkochen, Germany) operating at $100 \mathrm{kV}$. Findings were documented with a side-mounted $2 \mathrm{k} \times 2$ k-CCD-camera (TRS, Moorenweis, Germany). The iTEM software (Olympus, Tokyo, Japan) was used to measure collagen fibril diameter. Five images (40000x magnification)/tendon $(n=2 /$ group/time point) were used for quantification, resulting in average of 2800 fibrils/group/time point being analyzed (Supplementary Table 2).

\section{Micro-computed tomography $(\mu \mathrm{CT})$}

Muscle-Achilles tendon-calcaneus complex (contralateral, non-injured control, and injured Achilles tendons) were excised and collected 100 days post-injury ( $n=7-8$ animals/genotype), fixed as described above and scans were performed with the $\mu C T$ system Phoenix v|tome|x s 240/180 (GE Sensing \& Inspection Technologies, Frankfurt am Main, Germany). The scanning parameters were as follows: $50 \mathrm{kV}$ voltage, $620 \mu \mathrm{A}$ current, $500 \mathrm{~ms}$ time, 2000 images, voxel size $10 \mu \mathrm{m}$. The 3D images, the surface area and volume parameters were obtained with the software Volume Graphics VG Studio Max 2.2.3 (Volume Graphics, Heidelberg, Germany). Detailed description provided in Supplementary information.

\section{Biomechanical testing}

Mouse hindlimbs were explanted, wrapped in PBS-soaked gauze and stored at $-20^{\circ} \mathrm{C}$ until testing. On the testing day, hindlimbs were thawed for 30 min at RT. The viscoelastic biomechanical tests were performed with day 100 specimens (non-injured, contralateral and injured, $n=8-14 /$ genotype). Biomechanical testing at day 21 was not carried out, because the scar tissue is dominated by cells and literature has previously reported that viscoelastic properties of rodent Achilles tendons are greatly compromised in first weeks after injury [28, 29]. A LM1 machine (TA Instruments, New Castle, USA) and custom-made clamps, securing the calcaneal and myotendinous junction ends, were used. All tests were performed in PBS-bath at RT. The testing protocol was based on Dourte et al. [30] and Hochstrat et al. [31]. Detailed description provided in Supplementary information. The analysis of the data was carried out with a custom-written Matlab software (MathWorks, Natick, Massachusetts, USA) protocol.

\section{Voluntary running tests}

Mice ( $n=7-10 /$ genotype) were acclimatized to the experimental cage, containing a standard free-spinning mouse running wheel $(12 \mathrm{~cm}$ diameter) equipped with wired bike computer BC 5.16 (Sigma Sport, Neustadt, Germany) for 3 days. At day 4, mice were placed individually in the experimental cage, left overnight $(12 \mathrm{~h})$ to voluntarily use the wheel and the running distance was recorded.

\section{Single cell RNA-Sequencing (scRNA-Seq)}

Control, non-injured Tnmd ${ }^{-1-}$ SCXGFP ${ }^{+}$and WT SCXGFP ${ }^{+}$Achilles tendons ( $n$ $=2$ ) were explanted and $\mathrm{GFP}^{+}$cells were isolated by collagenase digestion $(8 \mathrm{~h})$ and filtration according to [22]. Next, cells ( $n=20 /$ genotype) were resuspended in PBS, placed on Adcell diagnostic slides (Thermo Fisher, Waltham, Massachusetts, USA), picked up in $1 \mu \mathrm{l}$ PBS each using a micromanipulator (Patchman NP2) with pump (CellTram, both Eppendorf, Hamburg, Germany) and subsequently stored in Smart-Seq2 lysis buffer at $-80^{\circ} \mathrm{C}$. The whole transcriptome amplification (WTA) and Illumina Nextera XT library preparation (Illumina, San Diego, California, USA) were performed as described by Picelli et al. [32]. The libraries were quantified using the KAPA Library Quantification kit (Roche Diagnostics, Mannheim, Germany), pooled in equimolar amounts and sequenced paired-end with read length of $2 \times$ $150 \mathrm{bp}$ and yield of 30 million reads per library on an Illumina HiSeq. In total, six ScxGFP ${ }^{+}$cells ( $n=6 /$ genotype) were subjected to scRNA-Seq. Bioinformatic analysis is described in Supplementary information.

\section{Statistical analysis}

Statistical calculations and graphs preparation were performed with GraphPad Prism 7 (San Diego, CA, USA). The results are presented as box plots (including dot plots where each dot represents an animal) with median and interquartile range (IQR), the whiskers show minimum and maximum values. After normality Shapiro-Wilk check, two-group analyses were performed, if not otherwise stated, with 2-tailed parametric unpaired Student's $t$-test. Multi-group comparisons were evaluated by one-way ANOVA with Bonferroni post-hoc test. Differences were considered statistically significant according to $p$-values of ${ }^{*} p<0.05$, ${ }^{* *} p<0.01$, ${ }^{* * *} p<0.001$ and ${ }^{* * * *} p<0.0001$. 


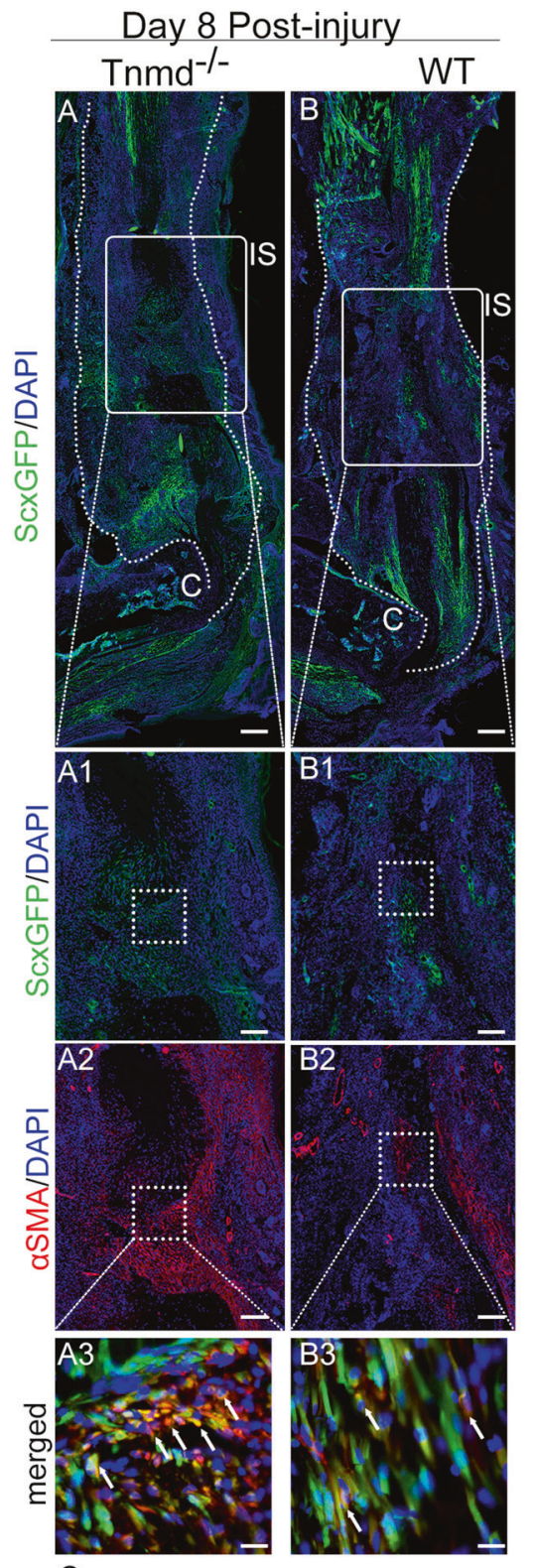

C

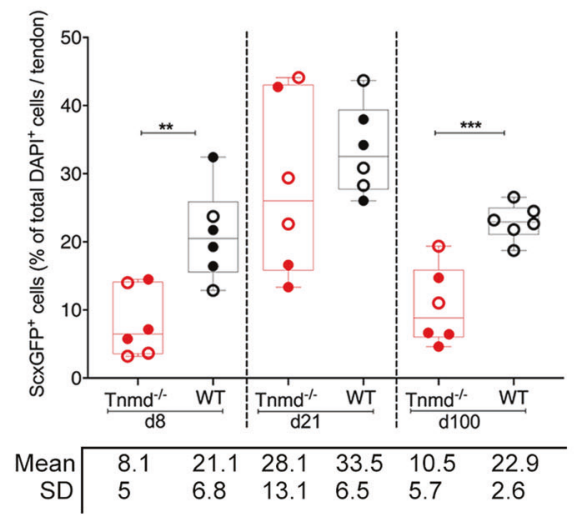

RESULTS

Tnmd $^{-/-}$scars harbor significantly higher number of aSMA cells and lower number of tendon-specific ScxGFP ${ }^{+}$cells during early and late tendon healing

Since ScxGFP ${ }^{+}$and aSMA ${ }^{+}$cells have been implicated as crucial players in the tendon repair process, we first investigated their
Day 21 Post-injury
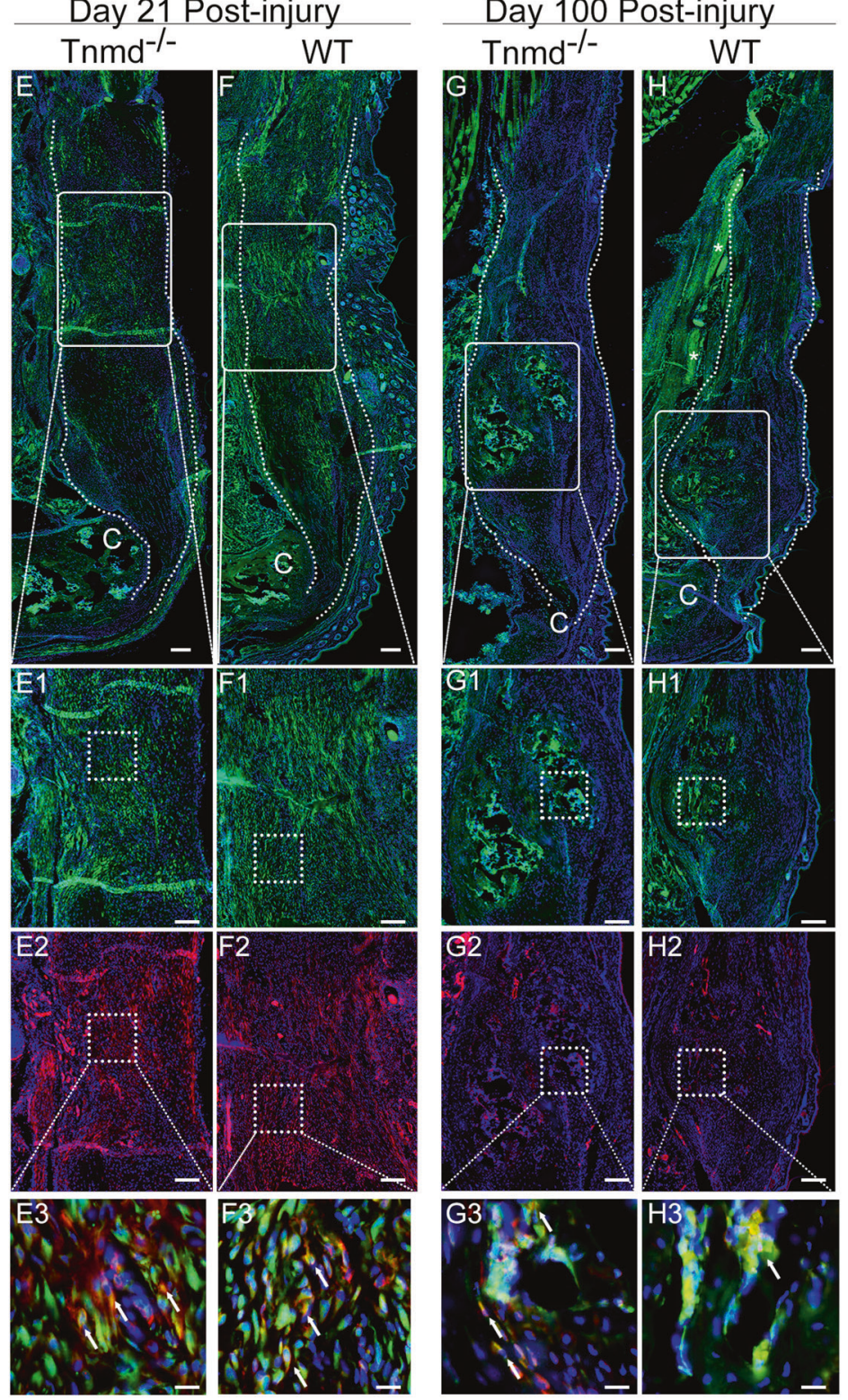

$\mathrm{D}$

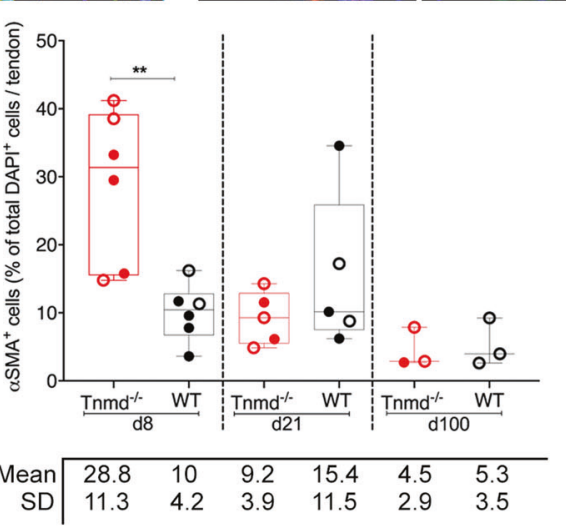

abundance by lineage tracing at day 8,21 and 100 post-injury. At the earliest time point $\mathrm{ScxGFP}{ }^{+}$cells were very scarce at the injury site (Fig. 1A-B1, C) and significantly less in the Tnmd $^{-1-}$ group. In contrast, approx. one third of $\mathrm{DAPI}^{+}$cells were positively stained for aSMA in Tnmd ${ }^{-1-}$ scars (Fig. 1A2, B2, D) compared to approx. one tenth in the WT (Fig. 1D). Of note, in the three examined time 
Fig. 1 Incidence of ScxGFP ${ }^{+}$and aSMA ${ }^{+}$cell populations during tendon healing at day 8, 21 and 100 post-injury. Representative images of injured Tnmd $^{-1-}$ ScxGFP and WT ScxGFP Achilles tendons at A, B day 8, E, F day 21 and $\mathbf{G}, \mathbf{H}$ day 100 post-injury. A1-H1 ScxGFP ${ }^{+}$cell occurrence over time. A2-H2 Corresponding immunofluorescence images of aSMA and merged images (A3-H3). White dotted lines frame Achilles tendons; white asterisks mark nerves; white arrows mark double positive ScxGFP ${ }^{+} / \alpha \mathrm{SMA}^{+}$cells. $\mathrm{C}=$ calcaneus, IS $=$ injury site. Scale bar: $200 \mu \mathrm{m}$ (panels A-F); $100 \mu \mathrm{m}$ (panels A1-H2); $20 \mu \mathrm{m}$ (A3-F3). Quantitative analysis of ScxGFP ${ }^{+}$(C) and $\alpha \mathrm{SMA}^{+}$(D) cells in percentage of total number of DAPI ${ }^{+}$cells within Achilles tendon scars. ScxGFP imaging, $\alpha$ SMA staining and quantification were performed with $n=3-6$ animals/genotype/time point; each animal represented by three tissue sections. Box plots show median \pm interquartile range (IQR), statistical significance was assessed with 2-tailed unpaired parametric Student's $t$-test, ${ }^{*} p<0.05,{ }^{* *} p<0.01$. Empty dot represents female mouse; filled dot represents male mouse.

points single cells were ScxGFP ${ }^{+}$and $\mathrm{aSMA}^{+}$(Fig. 1A3-H3). At day $21, \mathrm{ScxGFP}^{+}$cell numbers were strongly increased and bridged the defect between the sutured Achilles tendons ends (Fig. 1E-F1). Furthermore, a clear decline in aSMA ${ }^{+}$cells was detected in the Tnmd $^{-1-}$ group (Fig. 1D, E2, F2). At day 100, ScxGFP ${ }^{+}$and $\mathrm{aSMA}^{+}$ cells were present in the scar regions that appeared to be ossified (Fig. 1G-H2) and the percentage of ScxGFP ${ }^{+}$cells was significantly lower in the Tnmd $^{-/-}$tendons compared to WT (Fig. 1C), whilst the very low abundant aSMA ${ }^{+}$cells were comparable between the groups (Fig. 1D). Altogether, these data demonstrate that loss of Tnmd results in myofibroblast-enriched early scar tissue and significantly lower content of tendon lineage ScxGFP ${ }^{+}$cells during early and late repair stages.

\section{At day 21 post-injury, Tnmd $^{-/-}$scars are characterized by larger chondrogenic template and higher number of pro- inflammatory macrophages, but lower $\mathrm{BrdU}^{+}$cell count}

At day 21, haematoxylin-eosin (HE) staining revealed the presence of a cartilage-like nodule adjacent to the enthesis, large blood vessels and nerve fibers running in the peritendinous space of the injured tendons (Fig. 2A, B). High magnification images showed chondrocytic cells (Fig. 2A1, B1), which was confirmed by type II collagen (COLII) deposition in the territorial ECM (Fig. 2C-D1). Quantitative analysis of the $\mathrm{COLII}^{+}$areas revealed a tendency of larger cartilage template in the $\mathrm{Tnmd}^{-1-}$ group (Fig. 2E). Nerves and blood vessels were validated and quantified by neurofilament protein-heavy chain (NEFH) and collagen type IV (COLIV) stainings, but no genotype differences were detected (Supplementary Fig. 2A-F). Histological scoring [27] (Supplementary Table 1) suggested a tendency of inferior total scores for mutant compared to WT tendons (Supplementary Fig. 2G), which was in line with broader collagen III (COLIII)-rich regions, indicative of immature ECM in the Tnmd $^{-1-}$ tendons monitored by Herovici staining (Supplementary Fig. 3A-B1). Collagen fiber arrangement analysis in the scar tissue revealed no apparent differences between Tnmd $^{-1-}$ and WT (Supplementary Fig. 3C-D1). Next, we evaluated the incidence of pericyte, multipotent progenitors by immunofluorescent staining for CD146, but no significant difference in the number of $\mathrm{CD}_{146}{ }^{+}$cells was detected between the genotypes (Supplementary Fig. 2H-J). Similarly to day 8 [26], at day 21 Tnmd $^{-1-}$ scars contained significantly less BrdU ${ }^{+}$cells (Fig. 2F, G), higher numbers of $\mathrm{CD}^{+} 8^{+}$macrophages (Fig. $2 \mathrm{H}-\mathrm{J}$ ) and less antiinflammatory $\mathrm{CD}_{163}{ }^{+}$macrophages (Fig. 2K-M). Lastly, the expression of 14 tendon-related gene markers (Supplementary Fig. 3E) was evaluated by qRT-PCR. The analysis revealed the significant downregulation of 9 of the genes, besides of Scx, in the $\mathrm{Tnmd}^{-1-}$ group, which might be indicative of delayed activation of tenogenesis in the scar (Supplementary Fig. 3F). In sum, the above data reveals that certain parameters were comparable between genotypes, but the absence of Tnmd in the proliferative stage of the tendon healing process leads to abnormal, rather proinflammatory macrophage profile and reduced cell proliferation suggesting overall soft tissue destruction favouring ectopic chondrogenesis.

\section{The ECM of Tnmd $^{-1-}$ tendons is distinguished by lower collagen fibril density concomitant with significantly thicker and atypically shaped collagen fibrils}

Tendon ECM ultrastructure was investigated by transmission electron microscopy (TEM) and quantitative analyses of collagen fibril diameter distribution. At day 21, representative TEM images of injured Tnmd ${ }^{-/-}$and WT Achilles tendons revealed hypercellularity, decreased fibril size and density, and broader gaps between cells and fibrils (Fig. 3A-B3) in comparison to the non-injured controls (Supplementary Fig. 4A-D1). Quantitative analyses showed that in the WT group the majority of the fibrils were clustered in the range of $10-50 \mathrm{~nm}$ diameters. In contrast, scattered and thicker collagen fibrils, reaching diameters of up to $340 \mathrm{~nm}$, with irregular rough outlines were significantly enriched in the $\mathrm{Tnmd}^{-1-}$ tendons (Fig. 3A3, B3, E and F). This pattern of collagen fibril shift towards significantly large sizes in $\mathrm{Tnmd}^{-1-}$ was consistent in the injured setting at day 100 (Fig. 3C-D3, E, and F), as well as in the Tnmd ${ }^{-1-}$ contralateral tendons from day 21 (6 month-old) and 100 (9 month-old) (Supplementary Fig. 4A2-D2, E). In the late post-injury stage mineral deposits within the ECM and osteocyte-like cells, a sign of $\mathrm{HO}$, were detected in both, Tnmd ${ }^{-1-}$ and WT injured tendons (Fig. 3C-D1). Taken together, the TEM imaging reproducibly demonstrated a $\mathrm{Tnmd}^{-1-}$-specific phenomenon, namely significant fibril thickening in both conditions, before and after injury, underpinning that Tnmd acts as a regulatory factor of collagen fibrillogenesis.

\section{Loss of Tnmd leads to significantly higher trauma-induced} heterotopic ossification of Achilles tendon

At the late repair stage (day 100), HE staining revealed that the cartilage template was replaced with prevalent bone-like tissue located proximally of the entheses (Fig. 4A). Moreover, histological scoring [27] (Supplementary Table 1) showed significantly inferior values for the mutant group (Fig. 4B). The most remarkable differences were regarding larger bone nodules containing trabecular space, poorer cell alignment in the fibrous regions of the tendon and looser ECM judged by faint Eosin staining. To exclude dystrophic calcification we performed immunofluorescence staining with the osteogenic marker osteopontin (OPN) (Fig. 4C-D1) which demonstrated broader $\mathrm{OPN}^{+}$domain and higher protein levels in the $\mathrm{Tnmd}^{-/-}$group. In the bone nodules, sparse double positive ScxGFP ${ }^{+} / \mathrm{OPN}^{+}$cells were visible (Fig. 4C2-D2"). Next, to precisely quantify if the $\mathrm{HO}$ was higher in the injured $\mathrm{Tnmd}^{-1-}$ tendons we employed $\mu \mathrm{CT}$ scanning and quantification. First, scans of contralateral and non-injured, control tendons showed in the 9-month old animals age-related occurrence of a scarce in-tendinous $\mathrm{HO}$, which was comparable between the genotypes (Supplementary Fig. 5A-D). With regards to the injured setting, longitudinal and cross-sectional representative images demonstrated a bipolar $\mathrm{HO}$ distribution in the tendon with the enthesial-adjacent site pervading the myotendinous junction (MTJ)-adjacent site (Fig. 4E). Total HO surface in the injured Tnmd $^{-1-}$ tendons was significantly higher than the WTs (Fig. 4F) and similar tendency was observed for total $\mathrm{HO}$ volume (Fig. 4G). The measured $\mathrm{HO}$ surface at the enthesis was significantly 


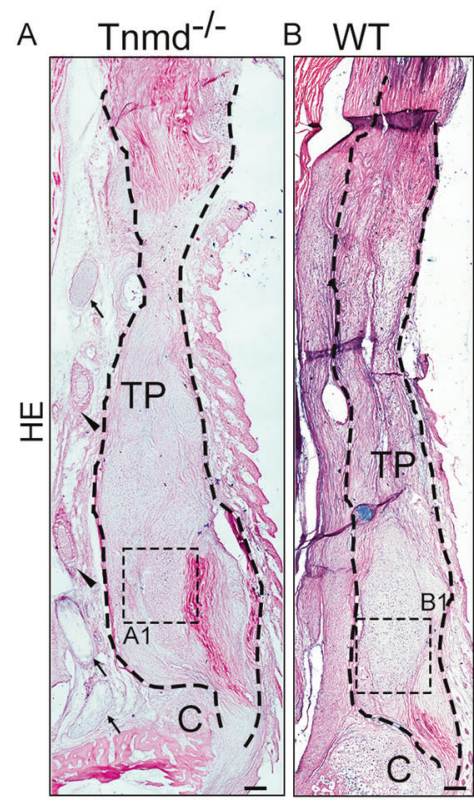

$\mathrm{E}$

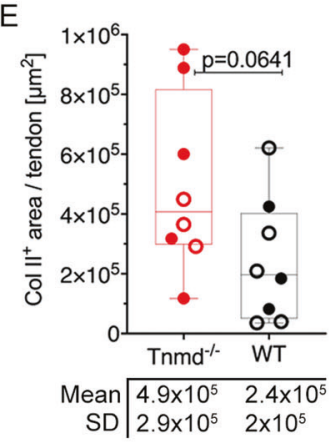

C $T$ Tnt

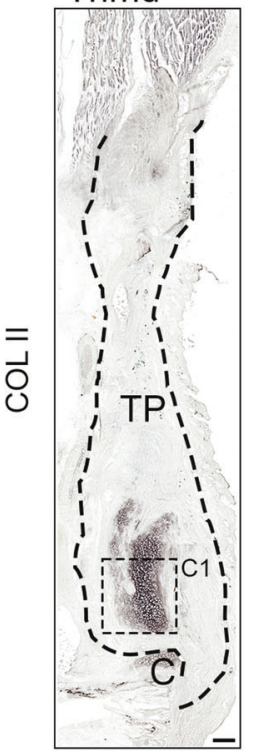

G

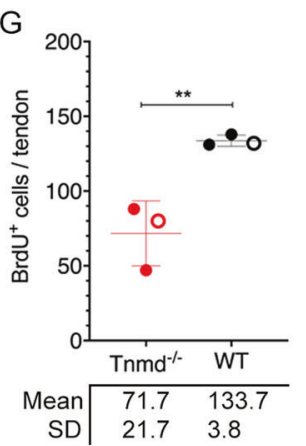

D WT

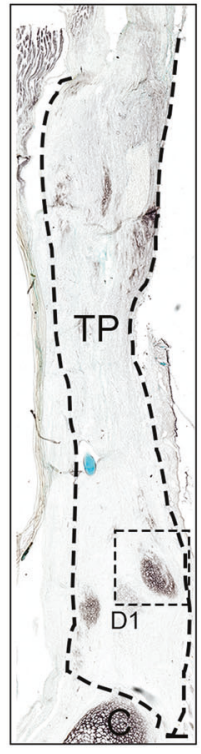

$\mathrm{J}$

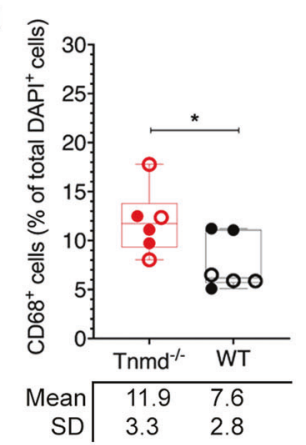

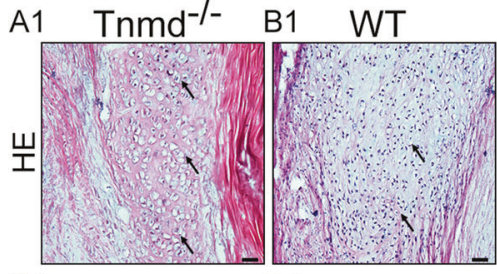
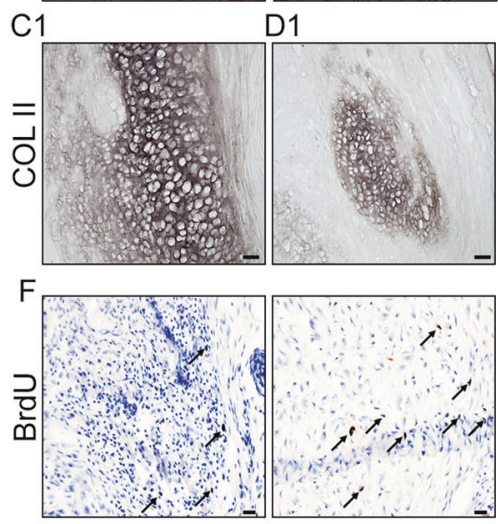

$\mathrm{M}$
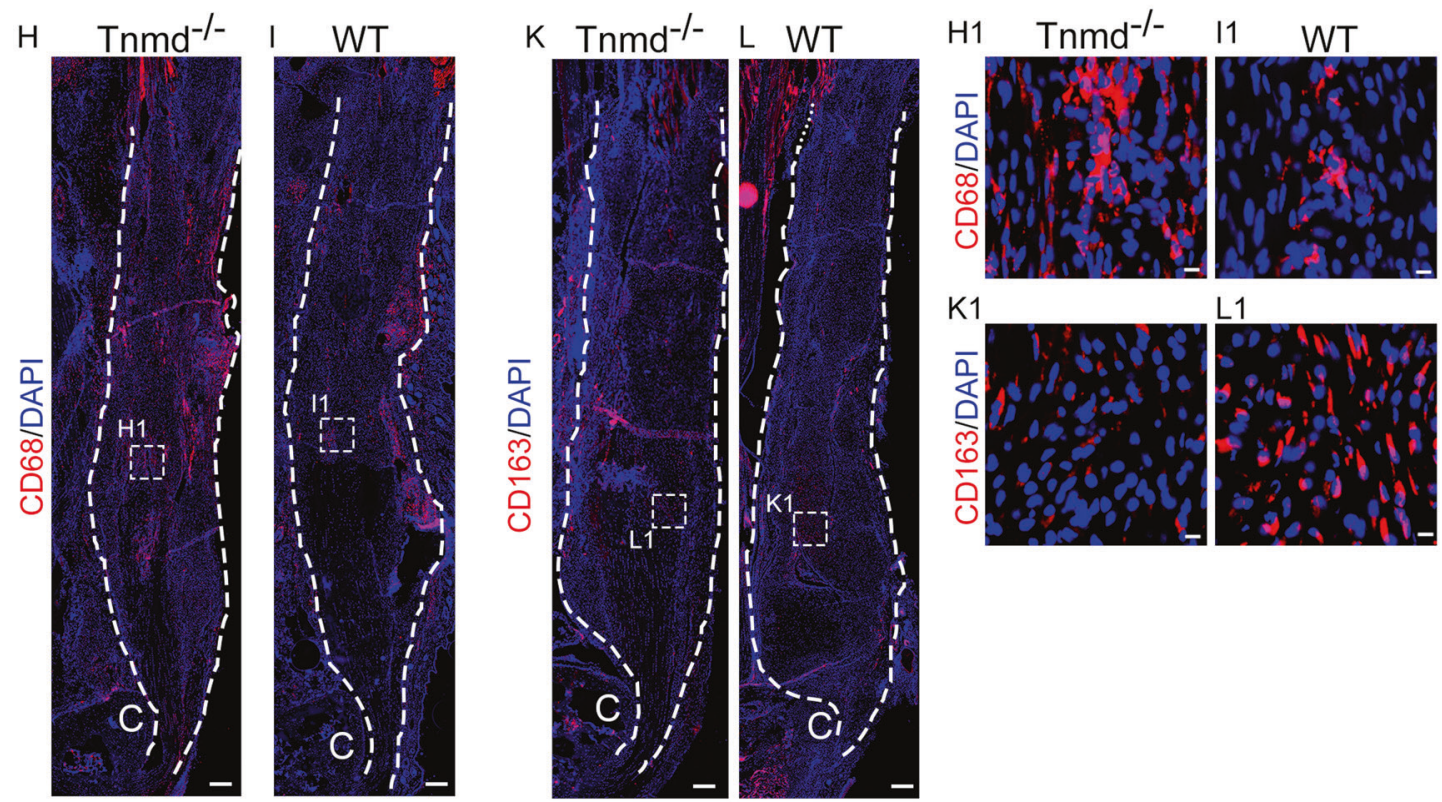

increased in Tnmd $^{-1-}$ injured tendons compared to WT (Fig. 4H), while the MTJ-adjacent $\mathrm{HO}$ surface was comparable between the genotypes (Fig. 4l). In order to disregard that Tnmd-deficiency affects bone quality, regional calcaneal and tibiofibular bone

volume and surface of specimens from the same groups were quantified and no significant differences were detected (Supplementary Fig. 5E-I). Lastly, qRT-PCR analysis revealed a significantly higher mRNA levels of the majority of assessed genes in $\mathrm{Tnmd}^{-/-}$ 
Fig. 2 Comparison of tendon repair between Tnmd $^{-1-}$ and WT mice at day 21 post-injury. A, B Representative mosaic HE and (A1, B1) higher magnification images. C, D Representative images and (C1, D1) higher magnification DAB images for type II collagen (COLII). E Quantification of $\mathrm{COLII}^{+}$area/tendon. F BrdU staining and $\mathbf{G}$ quantification of $\mathrm{BrdU}^{+}$cells/tendon. $\mathbf{H}$, I Representative fluorescence images for CD68, $(\mathrm{H} 1,11)$ higher magnification images and $\mathbf{J}$ quantification of $\mathrm{CD}^{+} \mathbf{8}^{+}$cells. $\mathbf{K}$, $\mathbf{L}$ Representative immunofluorescence staining for CD163, $(K 1, L 1)$ higher magnification images and $\mathbf{M}$ quantification of $\mathrm{CD}_{163^{+}}$cells. Black and white dotted lines frame Achilles tendons; vessels and nerves in the peritendinous space are marked with black arrowheads and arrows, respectively; blue bright spot in B and D: cerclage 6-0 prolene suture material; black arrows in (F) mark BrdU ${ }^{+}$cells. $C=$ calcaneus, TP = tendon proper. Scale bar: $200 \mu \mathrm{m}$ (panels A-L); $50 \mu \mathrm{m}$ (panels A1-D1); $20 \mu \mathrm{m}$ (panels F, H1-L1). Stainings and quantifications were performed with $n=3-8$ animals/genotype; each animal represented by three mosaic tissue sections. Box plots show median \pm IQR; statistical significance was assessed with 2-tailed unpaired parametric Student's $t$-test, ${ }^{*} p<0.05,{ }^{* *} p<0.01$. Empty dot represents female mouse; filled dot represents male mouse.

tendons (Supplementary Fig. 3G), which might be indicative of still ongoing tenogenesis. Hence, these results report for the first time that lack of Tnmd causes overall worsening of the late stage of tendon repair process coupled with significantly augmented trauma-induced $\mathrm{HO}$.

\section{Tnmd $^{-1-}$ tendons are naturally stiffer and display significantly increased static and dynamic Elastic moduli; post-injury, tendon viscoelastic properties are profoundly weakened in both genotypes}

We subjected non-injured, control and injured $\mathrm{Tnmd}^{-/-}$and WT Achilles tendons to a viscoelastic biomechanical testing at day 100 post-injury (Supplementary Fig. 6A, B: testing apparatus and protocol, and $6 \mathrm{C}-\mathrm{F}$ representative force-displacement curves). Cross-sectional tendon areas were measured and significantly larger values were obtained for the injured tendons compared to the controls, but no significant differences were detected between the genotypes (Fig. 5A). Next, dynamic Elastic modulus was measured at 3 different strain levels (4\%,6\% and 8\%) revealing significantly higher values at each deformation level in control Tnmd $^{-1-}$ tendons compared to WT (Fig. 5B). Upon injury, dynamic E-modulus decreased considerably compared to non-injured controls, but a genotype difference was still detectable at $8 \%$ strain (Supplementary Fig. 6G). Furthermore, we evaluated the static E-modulus and yet again, the values in control Tnmd $^{-1-}$ tendons were significantly higher than in the WT. At day 100 postinjury, this parameter showed significant and comparable 2-fold decrease in both genotypes (Fig. 5C). We also measured the load required to produce tissue failure, and found a significant difference towards lesser load inducing failure in the injured groups, but our biomechanical system did not detect significant changes between the control groups (Supplementary Fig. $6 \mathrm{H}$ ). Lastly, similar to the static E-modulus, control $\mathrm{Tnmd}^{-1-}$ tendons exhibited a strong tendency $(p=0.0543)$ to be stiffer than WT tendons (Fig. 5D). Irrespective of genotype, injury led to significant loss of tissue stiffness at day 100 that might relate to the observed trauma-induced tendon $\mathrm{HO}$ at this stage. Because of the significantly impaired tendon biomechanical properties upon injury, as well as the differences in the contralateral Achilles tendons, we tested the tissue functionality by allowing Tnmd $^{-1-}$ and WT mice to voluntarily undertake overnight running at day 100 post-injury (Fig. 5E). We detected a significantly lower running distance in Tnmd $^{-1-}$ animals, which we attributed to their significantly stiffer and hence inefficient contralateral tendons compared to WTs (Fig. 5F). Altogether, we demonstrate a profound decline in tendon viscoelastic properties at the late stage of Achilles tendon healing concomitant with diminished running behavior of the $\mathrm{Tnm}^{-1-}$ group; moreover, non-injured Tnmd-deficient tendons are stiffer and exhibit increased resistance to elastic deformation and reduced capacity to store musclegenerated energy. All in all, in Fig. $5 \mathrm{G}$ we provide a graphical synopsis of the major phenotypic changes in Tnmd-deficient mice over the time course after injury.
Pilot single cell RNA-sequencing analysis reveals a significant transcriptomic shift between Tnmd $^{-/-}$SCXGFP ${ }^{+}$and WT ScxGFP ${ }^{+}$cells

To fish out potential transcriptomic alterations driven by the absence of Tnmd in tendon lineage cells, we subjected Tnmd $^{-1-}$ $\mathrm{ScxGFP}^{+}$and WT ScxGFP ${ }^{+}$cells, isolated from non-injured Achilles tendons, to single cell RNA-sequencing (scRNA-seq) analysis. A total of 1278 significantly differentially expressed genes (DEGs) were identified between the genotypes by DESeq2-analysis. Volcano plot revealed that 529 DEGs were upregulated and 749 DEGs were downregulated in the Tnmd $^{-1-}$ cells compared to the WTs (Fig. 6A). Heat map (Fig. 6B) of the top 100 significant DEGs pointed out gene targets such as ninjurin-1 (Ninj1), E2F-associated phosphoprotein (Eapp), reticulocalbin-3 (Rcn3), serpin H1 (Serpinh 1), spinlin-1 (Spin 1) and secreted protein acidic and cysteine rich (Sparc), to be significantly downregulated in $\mathrm{Tnmd}^{-/}$group. These genes are linked to regulatory mechanisms of cell adhesion, proliferation, apoptosis and collagen synthesis. In contrast, cyclin-D-binding Myb-like transcription factor 1 (Dmtf1), E3 ubiquitin-protein ligase Rnfl130 and Rnfl167, glycosaminoglycan xylosylkinase (Fam2Ob) and the constitutive coactivator of PPAR-gamma-like protein 1 (Fam120b) were significantly upregulated in Tnmd ${ }^{-1-}$ cells (Fig. 6B, Supplementary Table 3). These genes play roles in negative regulation of cell cycle, senescence, proteoglycan expression and adipose cell differentiation. Gene ontology (GO) analysis (Fig. 6C-E) of only the significantly DEGs showed enrichment of gene clusters under biological process (BP), molecular function (MF) and cellular component (CC). For BP, the top 10 enriched terms included "signaling", "cell surface receptor signaling pathway", "cell adhesion", "cell communication" and "circulatory system development" (Fig. 6C). For MF (Fig. $6 \mathrm{D})$, "protein domain specific binding", "phosphotransferase activity" and "purine and adenyl nucleotide binding" scored in the top 10 enriched terms. For CC, terms related to "cell periphery", "plasma membrane bounded cell projection" and "anchoring and cell junction" were among the top 10 enriched terms (Fig. 6E). When subjecting all DEGs to Kyoto Encyclopedia of Genes and Genomes (KEGG) signaling pathway analysis, hits on "Hippo and Wnt-signaling cascades", "metabolic and insulin signaling pathways", and "chemokine and receptor signaling pathways" were uncovered (Fig. 6F). In sum, this very novel scRNA-seq data demonstrates that loss of Tnmd results in profound gene expression changes in tendon lineage cells from the non-injured Achilles tendon setting. It also suggests unprecedented signaling pathways involving indirectly or directly Tnmd and strongly urges for follow up analyses on tendon lineage cells from the injured setting.

\section{DISCUSSION}

$\mathrm{HO}$ is an aberrant repair process involving ectopic bone formation in response to tendon tissue trauma [16]. First, local tissue destruction and inflammation are initiated followed by activation and proliferation of fibroblastic cells, which in turn can 
Day 21 Post-injury
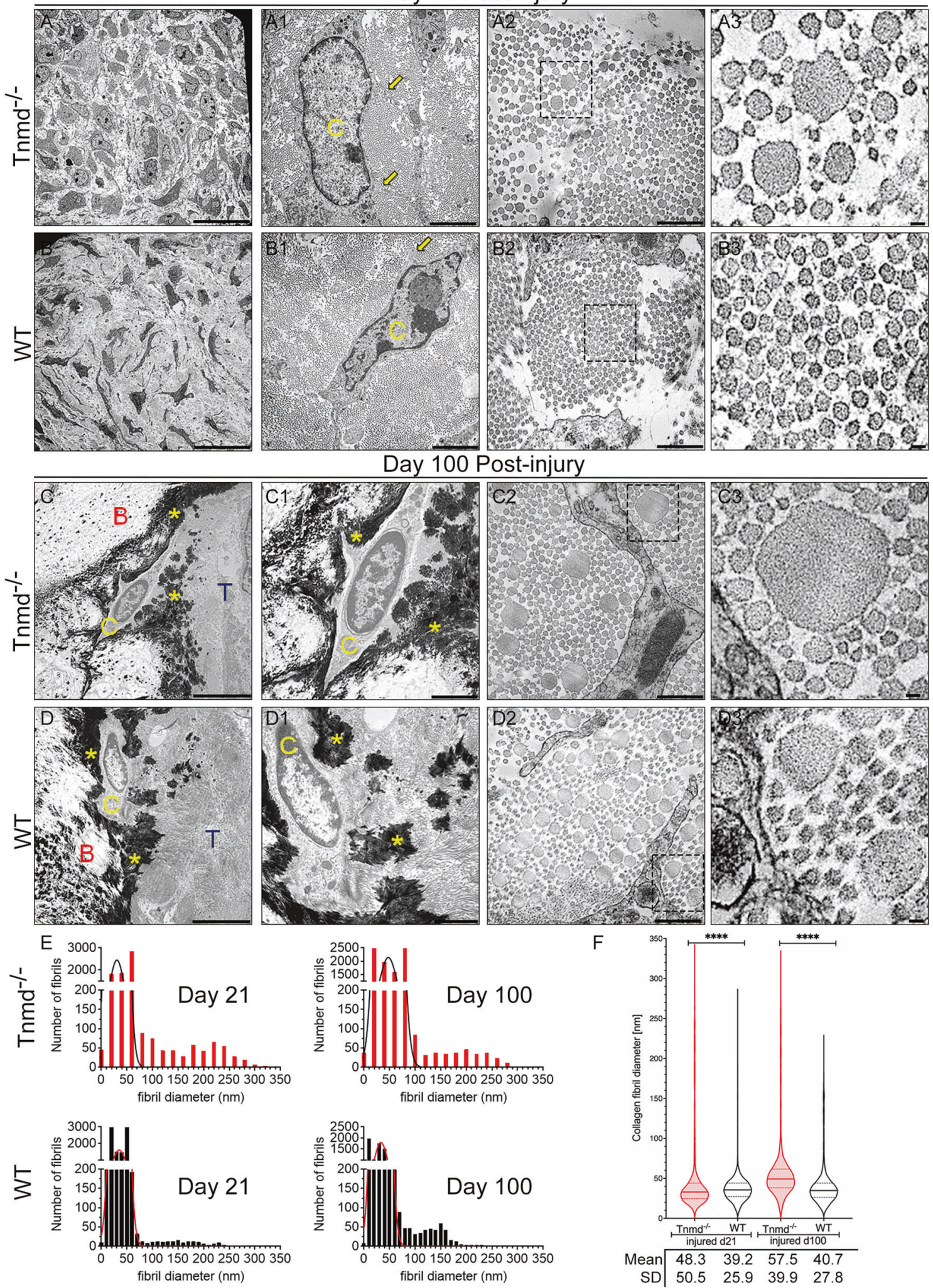

Fig. 3 Transmission electron microscopy and collagen fibril diameter analysis of Achilles tendons at day 21 and 100 post-injury. A-D Representative 2000x, A1-D1 10000x and A2-D3 40,000x magnification images of injured Tnmd ${ }^{-1-}$ and WT Achilles tendons at day 21 and 100 post-injury. $\mathrm{C}=$ cell; $\mathrm{B}=$ bone; $\mathrm{T}=$ tendon; yellow arrow = cell protrusions; yellow asterisk = mineralized zones. Scale bars: $20 \mu \mathrm{m}$ (panels $\mathbf{A}, \mathbf{B})$; $5 \mu \mathrm{m}$ (panels C, D); $2 \mu \mathrm{m}$ (A1-D1), $500 \mathrm{~nm}$ (panels A2-D3). E Histograms showing incidence of collagen fibril sizes. F Violin plot for collagen fibril diameter distribution, median $\pm \mathrm{IQR}$, statistical significance was calculated by 2-tailed unpaired nonparametric Mann-Whitney test, ${ }^{*} p<0.05,{ }^{* *} p<0.01,{ }^{* *} p<0.001,{ }^{* * *} p<0.0001$. Day $21, n=2$ animals/genotype; day $100, n=2$ animals/genotype. 

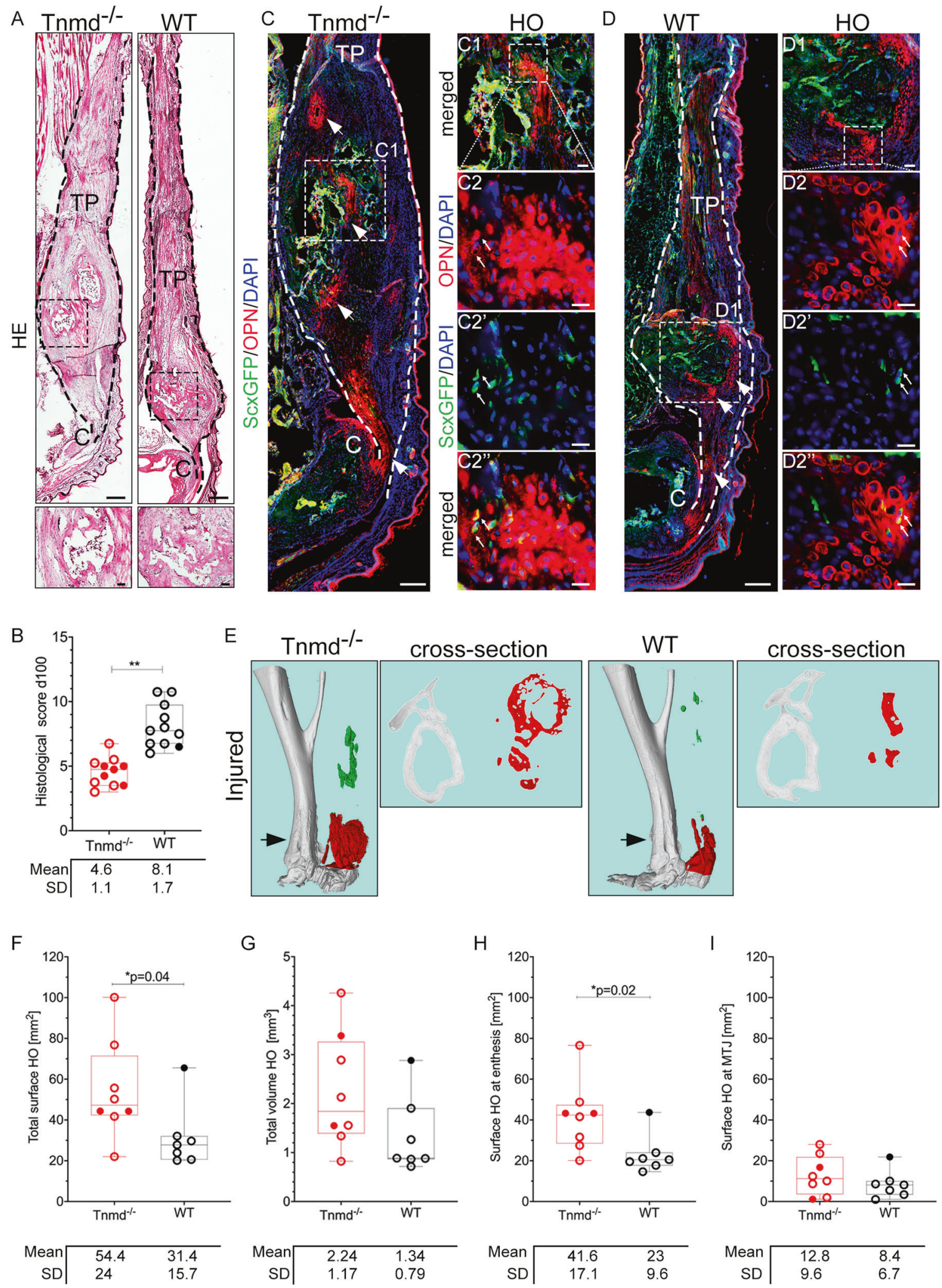

differentiate into chondrocytes and osteoblasts, thereby resulting in $\mathrm{HO}$ with adverse effects to the tendon structure, strength and function [33]. Despite the clinical relevance of trauma-induced tendon $\mathrm{HO}[3,4]$, the evolution of cell populations as well as the molecular mechanisms of $\mathrm{HO}$ have not been fully elucidated. Here,

we challenged Tnmd-knockout mice with complete Achilles tenotomy and we hypothesized Tnmd to be a protective factor of trauma-induced $\mathrm{HO}$ in tendon based on: (i) loss of Tnmd results in augmented fibrovascular scar tissue during early tendon healing [26]; (ii) Tnmd-deficiency is associated with aging-related 
Fig. 4 Comparison of tendon repair between Tnmd $^{-1-}$ and WT mice at day 100 post-injury. A Representative HE mosaic images (bottom panel, higher magnification). B Histological scoring at 100 days post-injury. C, D Representative fluorescent images and C1-D2" higher magnification images of Osteopontin (OPN)-staining of Tnmd ${ }^{-1-}$ ScxGFP and WT ScxGFP tendons. Black and white dotted lines frame Achilles tendons; white arrows in C, D mark OPN ${ }^{+}$-stained regions. White arrows in C2-D2" mark double positive $S c x G F P^{+} / \mathrm{OPN}^{+}$cells. C $=\mathrm{calcaneus}$ TP = tendon proper. Scale bar: $200 \mu \mathrm{m}$ (A panel); $100 \mu \mathrm{m}$, (C, D panels); $50 \mu \mathrm{m}$ (C1, D1 panels); $20 \mu \mathrm{m}$ (C2-D2" panels). E Representative longitudinal and cross-sectional $\mu \mathrm{CT}$ scans at day 100 post-injury. Quantification of total $\mathrm{HO}$ surface and volume (F, G), HO surface at the enthesis (H), and MTJ (I) in Achilles tendons 100 days post-injury. Histological scoring and $\mu C T$ was performed with $n=7-11$ animals/ genotype. OPN staining was performed with $n=3$ animals/genotype, each animal represented by three tissue sections. Box plots show median \pm IQR; statistical was evaluated by 2-tailed unpaired parametric Student's $t$-test for histological scoring, and unpaired non-parametric Mann-Whitney $U$ test for $\mu C T$ analysis, ${ }^{*} p<0.05,{ }^{* *} p<0.01$. Empty dot represents female mouse; filled dot represent male mouse.

mineralization of the chordae tendineae cordis [34]; and (iii) double knockout of Tnmd and its homolog chondromodulin I $(\mathrm{Chm} 1)$ leads to ectopic appearance of hypertrophic chondrocytes and osteoblasts in intervertebral disc [35].

Our results demonstrated that the absence of Tnmd alters the interplay between ScxGFP ${ }^{+}$tendon lineage cells and aSMAexpressing myofibroblastic cells. We detected 8 days after injury only minor numbers of ScxGFP ${ }^{+}$cells being located in the scar lesions, which is consistent with other studies [12, 36]. During the later repair stages, ScxGFP ${ }^{+}$cells increased although their numbers were significantly less in Tnmd ${ }^{-1-}$ than WT scars. Tnmd might directly influence the tendon lineage cells, since it has been shown to regulate tendon cell density, proliferation and senescence in vivo and in vitro $[20,22,26]$. Interestingly, we detected significantly more aSMA ${ }^{+}$cells in day $8 \mathrm{Tnmd}^{-1-}$ scars. In healthy tendons, aSMA ${ }^{+}$cells are contained in the vascularized epitenon sheaths [37], and upon injury they activate and dominate the fibrotic scar $[12,38]$. We propose that the significant influx of aSMA $^{+}$cells in Tnmd $^{-1-}$ scars is related to the enhanced vascularization of the ruptured mutant tendons [26]. Remarkably, we detected some double positive $\mathrm{ScxGFP}^{+} / \mathrm{aSMA}^{+}$cells in the three different time points post-injury. Best and Loiselle proposed that $\mathrm{Scx}^{+}$cells appear early in the scar tissue of injured and sutured tendons, but do not differentiate into myofibroblasts during healing [14] while several studies have suggested aSMA cell plasticity [39-42] including towards ScxGFP ${ }^{+}$[13]. Next, in day 21 scars we detected cartilaginous anlage with a tendency to be larger in the $\mathrm{Tnmd}^{-1-}$ group. The chondrocyte origin is still disputable and several cell types can differentiate into this lineage, including Scx-expressing subpopulations: $\mathrm{Scx} \mathrm{CD}^{+} 105^{-}$, $\mathrm{Scx}^{+} \mathrm{aSMA}^{+}, \mathrm{Scx}^{+} \mathrm{S} 100 \mathrm{a}^{+}{ }^{+}, \mathrm{Scx}^{+} \mathrm{Sox}^{+}$and Cathepsin- ${ }^{+}$cells [43-46]. Whether these discrete subpopulations have been altered upon Tnmd loss should be addressed in follow up research. Macrophages have a pivotal task in both stimulating and resolving inflammation, therefore assisting and regulating the ongoing tissue repair [7]. The phenotype reported at day 8 [26] was persistent also at day 21, suggesting that Tnmd absence can result in a chronic inflammation in the injured tendons. A study by Sorkin et al. revealed in burn tenotomy that invading macrophages drive tendon $\mathrm{HO}$ by producing transforming growth factor beta-1 (TGF-ß1), thus stimulating mesenchymal progenitors to chondrogenically differentiate [16]. Indeed, at day 8 significantly Tgf- $B 1$ mRNA levels were increased in tendon tissue extracts from $\mathrm{Tnmd}^{-1-}$ group [26]. The molecular mechanisms involved in the crosstalk between distinct cell populations during trauma-induced tendon $\mathrm{HO}$ still remains to be unraveled. To this end, our data provides the first evidences that Tnmd might be an important molecular factor involved in the interplay between cellular populations during tendon $\mathrm{HO}$ process.

We followed the repair process up to day 100 after injury, and we monitored ECM fibrillar composition by TEM, $\mathrm{HO}$ spreading by $\mu C T$ and biomechanical properties by viscoelastic testing. Our TEM data validated a very consistent ECM phenotypic feature specific to the loss of Tnmd [20,24], namely a significant increase of COL fibril diameters coupled with aberrant fibril morphology. At the ultrastructural level mineral deposits and osteocyte-like cells were observed in both genotypes at day 100 post-trauma; however, $\mu \mathrm{CT}$-based quantification revealed a significantly higher $\mathrm{HO}$ in Tnmd $^{-1-}$ tendons compared to WT. Biomechanical analysis showed that even after 100 days of injury, the tendons in both groups suffer significant negative changes in their viscoelastic properties compared to non-injured tendons. We detected only significant differences in injured tendons in the dynamic E-modulus at $8 \%$ strain, which might be related to the sensitivity of the biomechanical testing system when HO-compromised tendons were measured. However, we discovered in non-injured controls as well as in the contralateral tendons of injured animals that the absence of Tnmd results in a significant increase in static and dynamic E-moduli, representing Achilles tendon stiffening. These findings correlate with the pathological thickening of $\mathrm{COL}$ fibrils [20,24] and increase in their nano-stiffness [24] as well as dysregulated expression of genes regulating COL fibrilogenesis in Tnm $^{-1-}$ tendons [26]. Biomechanical alteration in the tendon tissue, serving as a spring in the locomotive apparatus can result in deficient storage and transmission of the muscle-generated energy, hence reduced tendon functionality. We have shown that Tnm $^{-/}$mice ran significantly less, experienced fatigue and developed secondary myopathy [24]. In this study, we subjected at day 100 post-injury Tnmd $^{-/-}$animals to voluntary running tests and found that, again, this group undertakes significantly shorter distances compared to the WTs. We propose that this behavior is the combined result of the significant stiffening of the contralateral Achilles tendons and significant $\mathrm{HO}$ of the injured ones.

Altogether, our results on the scar tissue composition and the data on qRT-PCR at day 8 [26], 21 and 100 can also be interpreted as the two study groups having an uneven healing baseline: the speed of the healing process in Tnm $^{-1-}$ group was delayed, while the WT group had more advanced scar remodeling. To clarify this hypothesis future investigations should assess time points later than 100 days to determine whether WTs reach full remodeling, while mutants maintain tendon $\mathrm{HO}$. Other relevant research approaches to consider are: evaluating an earlier cerclage removal thus enabling sooner load transmission; allowing animals to run in order to facilitate further remodeling; utilizing Tnmd overexpression mouse model to assess if healing can accelerate; and employing a commercially available recombinant Tnmd protein as a rescue trial.

Due to the lack of knowledge on direct binding partners of Tnmd, a major limitation of our study is the explanation of the molecular mode-of-action of this protein. Our scRNA-Seq analysis revealed clear genotype differences and suggested that loss of Tnmd results in dysregulated gene expression associated with cell adhesion, proliferation, senescence and collagen synthesis, the latter based on the significant downregulation of Rcn3 and Serpinh1 $[47,48]$. Among the enriched GO clusters, the frequently referenced genes belong to bone morphogenic protein family. KEGG signaling pathway analysis identified Hippo-signaling pathway, connected to the transcriptional co-activator complex YAP/ TAZ that plays key roles in tissue homeostasis and regeneration. Remarkably, elevated BMP- and Hippo-related signaling have been strongly allied with $\mathrm{HO}$ of mesenchymal progenitors [49]. Another KEGG hit was Wnt-pathway, which is in line with in vitro studies reporting that $\mathrm{Wnt} /$ B-catenin signaling can mediate Scx- 

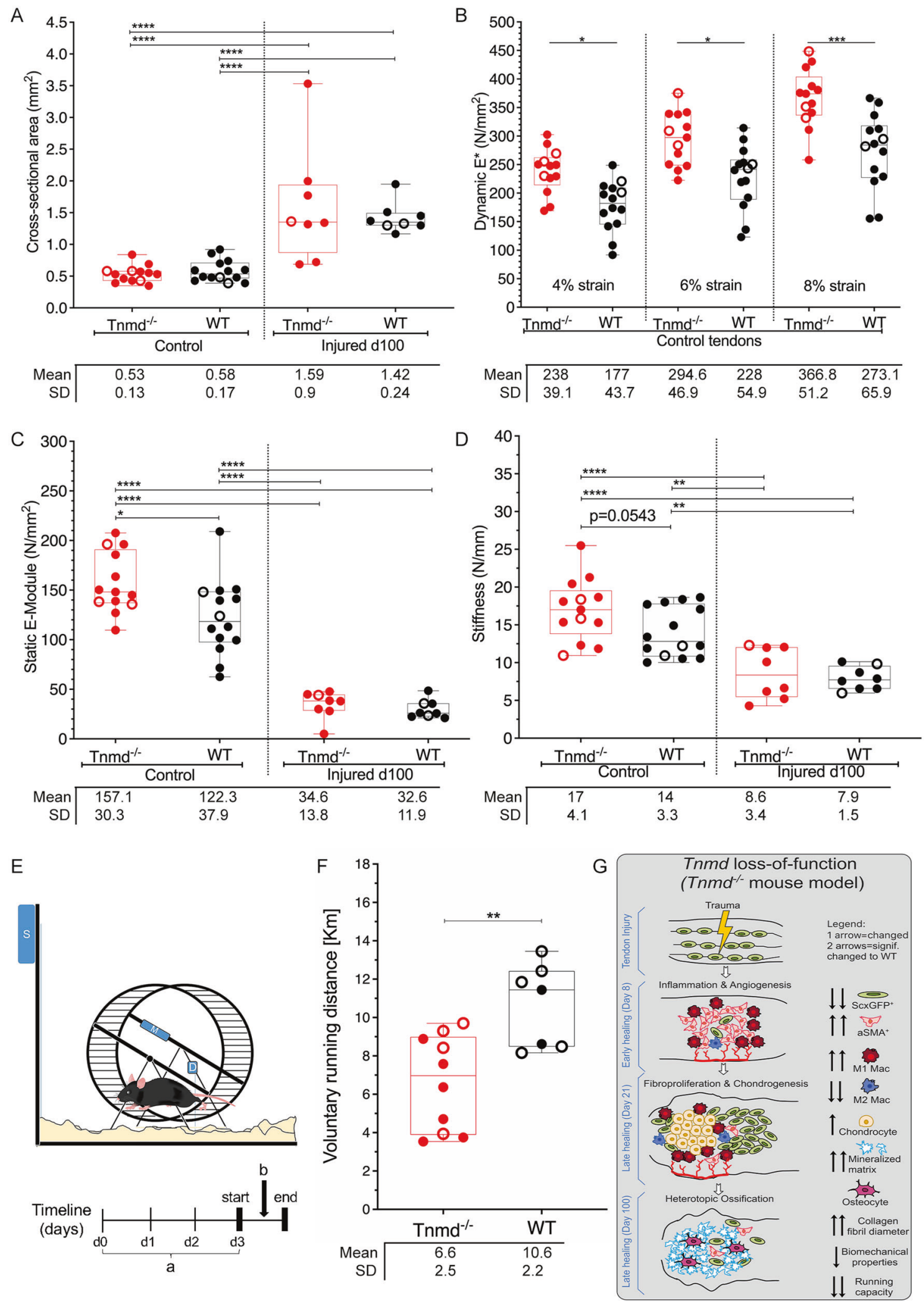

Fig. 5 Viscoelastic biomechanical testing of non-injured, control and injured Achilles tendons, and voluntary running tests at day 100 post-injury. A Cross-sectional area of non-injured, control and injured Achilles tendons. B Dynamic Elastic modulus in control Achilles tendons at $4 \%, 6 \%$ and $8 \%$ strain. C Static Elastic modulus and D stiffness of non-injured, control and injured Achilles tendons. Biomechanical testing was performed with $n=8-14$ animals/genotype. Box plots show median \pm IQR; statistical analysis was performed with one-way ANOVA and Bonferroni post-hoc test. E Test setup (upper drawing) and timeline (bottom drawing) for voluntary running tests. $\mathbf{F}$ Recorded running distance; tests were performed with $n=7-10$ animals/genotype. Box plots show median \pm IQR; statistical significance assessed with 2-tailed unpaired parametric Student's $t$-test, ${ }^{*} p<0.05,{ }^{* *} p<0.01$, ${ }^{* * *} p<0.001,{ }^{* * *} p<0.0001$. Empty dots represent female mouse; filled dots represent male mouse. G Graphical synopsis of the major phenotypic changes in $\mathrm{Tnmd}^{-/-}$animals over the time course after injury. 
A

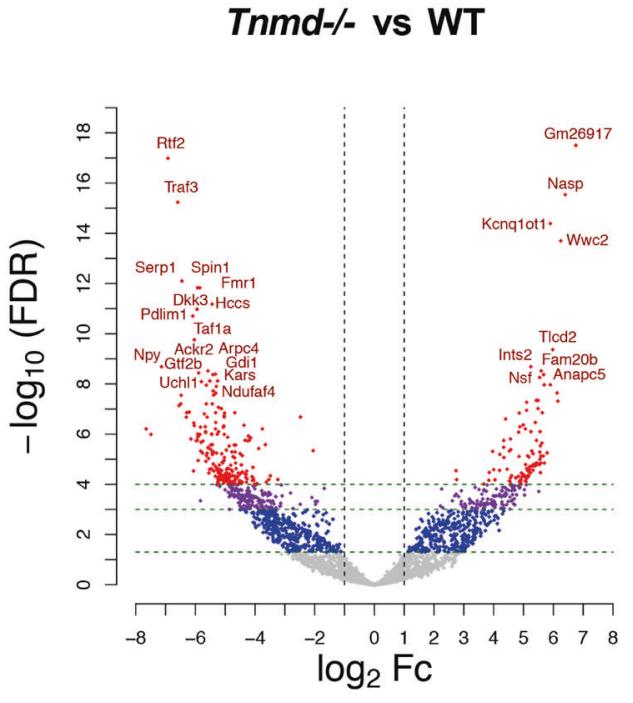

C Gene ontology: Biological process

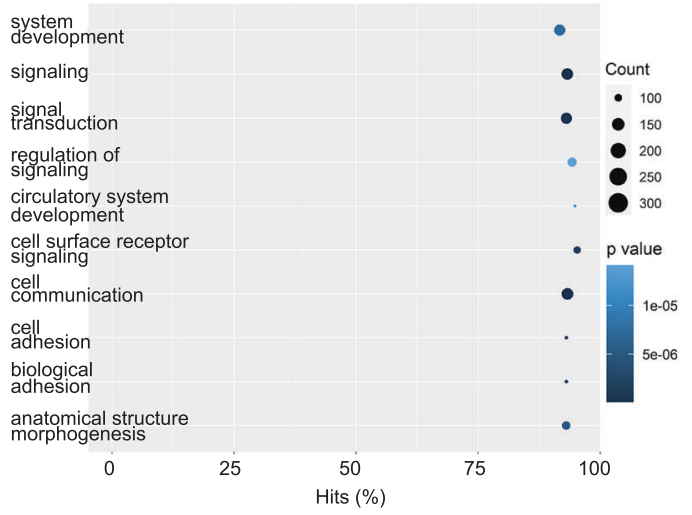

E Gene ontology: Celular components

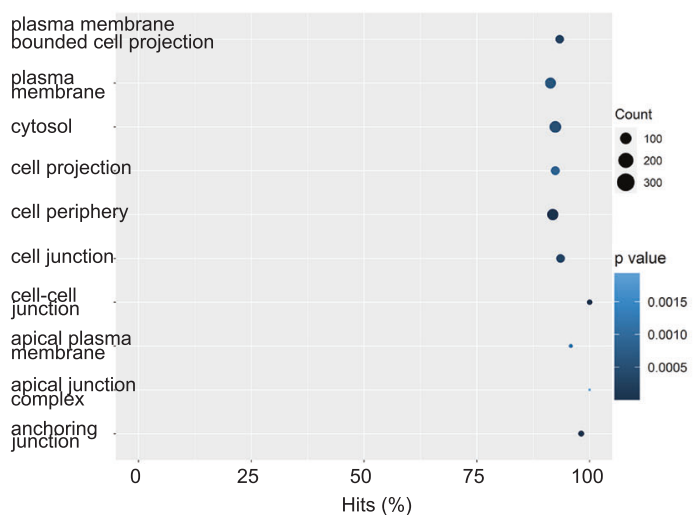

independent expression of Tnmd in tendon- and bone marrowderived mesenchymal stem cells [50, 51]. KEGG analysis also referenced metabolic and insulin pathways, which is in agreement with genetic, clinical and experimental studies that have linked Tnmd to metabolic syndrome, diabetes and obesity [19]. In all, the scRNA-seq approach can support the identification of putative Tnmd-dependent cascades and elucidation of Tnmd
B
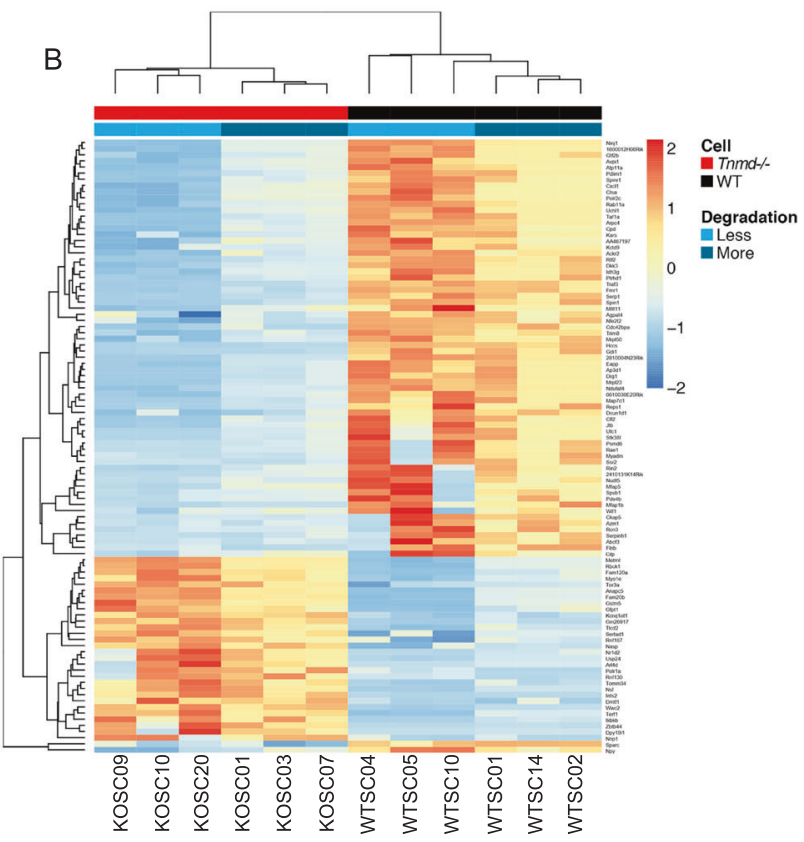

D

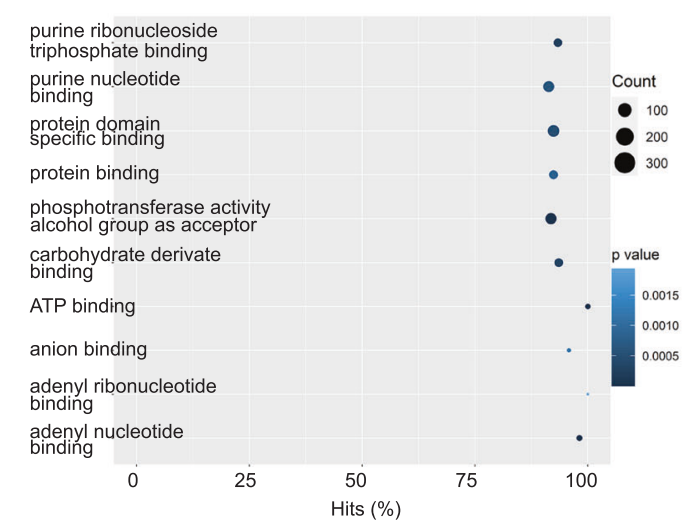

F KEGG pathway enrichment with selected terms

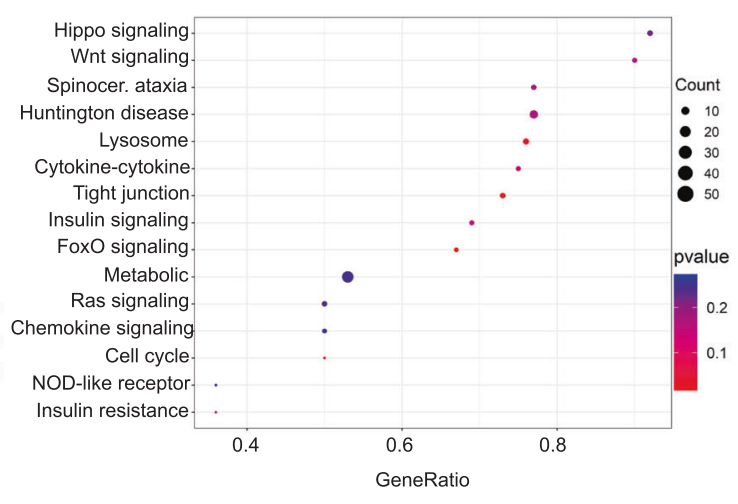

mode-of-action; hence, follow up studies should aim to screen and validate possible molecular targets and pathways as well as implement protein-protein interaction assays. Moreover, by employing scRNA-seq on tendon lineage cells isolated from distinct stages of the repair process, a better understanding of the longitudinal molecular "evolution" in the Achilles tendons from both genotypes can be gained. 
Fig. 6 Single cell RNA-sequencing of Tnmd $^{-1-}$ ScxGFP $^{+}$and WT ScxGFP ${ }^{+}$cells isolated from non-injured Achilles tendons. A Volcano plot shows $-\log _{10} F D R$ i.e. adjusted $p$-value and $\log _{2} F c$ expression values of all genes, each dot represents a gene. Significantly differentially expressed genes (DEGs) (- $\log _{10} \mathrm{FDR}<0.05$ and $\left.\left|\log _{2} \mathrm{FC}\right|>1\right)$ are highlighted in color other than gray. Vertical lines present the Log ${ }_{2} \mathrm{Fc}$ threshold equaling 1 or -1 ; genes outside these lines are DEGs with at least 2-Fc. The horizontal lines show -Log ${ }_{10}$ FDR corresponding to FDR thresholds of $0.05,0.001$ and 0.0001 from the bottom respectively. Red, purple and blue dots represent the significant DEGs with 2-Fc and $-\log _{10} \mathrm{FDR}<0.0001,-\log _{10} \mathrm{FDR}<0.001$ and $-\log _{10} \mathrm{FDR}<0.05$ respectively. Gray dots are neither significant nor have expression above 2-Fc. B Heat map depicting the $\log _{2}$ CPM expression value of selected top 100 significant DEGs. Top 100 genes with lowest q-value are depicted. Genes are colored according to their Log $_{2} \mathrm{CPM}$ expression values. Clustering on the top is based on cell type and degradation level. C-E GO enrichment analysis of the significantly DEGs; results are depicted in dot plot format where top 10 enriched ontology terms are listed on $y$-axis and the percentage of hits (number of DEGs/number of background genes associated to each term) are indicated on x-axis. Dots size represents the count of DEGs associated with each term, whilst the color shows the enrichment $p$-value. Top enriched terms from $C$ Biological Process, D Molecular Function and E Cellular Component. F KEGG signaling pathway analysis of the significant DEGs in dot plot format; $y$-axis shows top 15 enriched pathways; $x$-axis indicates gene ratio (number of DEGs/number of background genes associated to each term). Dots size is based on the number of DEGs that were associated with each term, whilst the color shows the $p$-value of enrichment.

Taken together, our study demonstrates that loss of Tnmd leads to long-term inferior tendon healing outcome characterized by: i) imbalance between tendon lineage and myofibroblastic cells during early stage; ii) persistent inflammatory macrophage profile and emergence of robust cartilaginous template during the proliferative stage; and iii) significant in-tendinous heterotopic ossification concurrent with compromised tendon viscoelastic properties and reduced running capacity at the remodeling stage (graphical synopsis, Fig. 5G). Thus, we report that Tnmd plays an important role during the course of tendon healing by alleviating trauma-induced $\mathrm{HO}$ and, therefore, is a suitable gene candidate for the generation of novel dual function therapeutic drugsaccelerating repair and reducing the risk of undesired ossification of injured tendons.

\section{DATA AVAILABILITY}

The authors declare that the datasets generated and/or analyzed during the current study are available from the corresponding author on reasonable request. The scRNASeq datasets used for the analysis have been deposited into the Gene Expression Omnibus database at the National Center for Biotechnology Information (Accession number GSE179454).

\section{REFERENCES}

1. Meyers C, Lisiecki J, Miller S, Levin A, Fayad L, Ding C, et al. Heterotopic ossification: a comprehensive review. JBMR. 2019;3:e10172.

2. Xu R, Hu J, Zhou X, Yang Y. Heterotopic ossification: mechanistic insights and clinical challenges. Bone. 2018;109:134-42.

3. Kraus R, Stahl J-P, Meyer C, Pavlidis T, Alt V, Horas U, et al. Frequency and effects of intratendinous and peritendinous calcifications after open Achilles tendon repair. Foot Ankle Int. 2004;25:827-32.

4. Ateschrang A, Gratzer C, Weise K. Incidence and effect of calcifications after open-augmented Achilles tendon repair. Arch Orthop Trauma Surg. 2008;128:1087-92.

5. Lin TWTW, Cardenas L, Soslowsky LJLJ. Biomechanics of tendon injury and repair. J Biomech. 2004;37:865-77.

6. Freedman BR, Rodriguez AB, Leiphart RJ, Newton JB, Ban E, Sarver JJ, et al. Dynamic loading and tendon healing affect multiscale tendon properties and ECM stress transmission. Sci Rep. 2018;8:10854.

7. Thomopoulos S, Parks WC, Rifkin DB, Derwin KA. Mechanisms of tendon injury and repair. J Orthop Res. 2015;33:832-9.

8. Agarwal S, Sorkin M, Levi B. Heterotopic ossification and hypertrophic scars. Clin Plast Surg. 2017;44:749-55.

9. O'Brien EJO, Frank CB, Shrive NG, Hallgrímsson B, Hart DA. Heterotopic mineralization (ossification or calcification) in tendinopathy or following surgical tendon trauma. Int J Exp Pathol. 2012;93:319-31.

10. Murchison ND, Price BA, Conner DA, Keene DR, Olson EN, Tabin CJ, et al. Regulation of tendon differentiation by scleraxis distinguishes force-transmitting tendons from muscle-anchoring tendons. Development. 2007;134:2697-708.

11. Pryce BA, Brent AE, Murchison ND, Tabin CJ, Schweitzer R. Generation of transgenic tendon reporters, ScxGFP and ScxAP, using regulatory elements of the scleraxis gene. Dev Dyn. 2007;236:1677-82.

12. Howell K, Chien C, Bell R, Laudier D, Tufa SF, Keene DR, et al. Novel model of tendon regeneration reveals distinct cell mechanisms underlying regenerative and fibrotic tendon healing. Sci Rep. 2017;7:45238.
13. Dyment NA, Hagiwara Y, Matthews BG, Li Y, Kalajzic I, Rowe DW. Lineage tracing of resident tendon progenitor cells during growth and natural healing. PLoS ONE. 2014;9:e96113.

14. Best KT, Loiselle AE. Scleraxis lineage cells contribute to organized bridging tissue during tendon healing and identify a subpopulation of resident tendon cells. FASEB J. 2019;33:8578-87.

15. Dyment NA, Liu C-F, Kazemi N, Aschbacher-Smith LE, Kenter K, Breidenbach AP, et al. The paratenon contributes to scleraxis-expressing cells during patellar tendon healing. PLoS ONE. 2013;8:e59944.

16. Sorkin M, Huber AK, Hwang C, Carson WF, Menon R, Li J, et al. Regulation of heterotopic ossification by monocytes in a mouse model of aberrant wound healing. Nat Commun. 2020;11:722.

17. Brandau O, Meindl A, Fässler R, Aszódi A. A novel gene, tendin, is strongly expressed in tendons and ligaments and shows high homology with chondromodulin-I. Dev Dyn. 2001;221:72-80.

18. Shukunami C, Oshima Y, Hiraki Y. Molecular cloning of tenomodulin, a novel chondromodulin-I related gene. Biochem Biophys Res Commun. 2001;280:1323-7.

19. Dex S, Lin D, Shukunami C, Docheva D. Tenogenic modulating insider factor: Systematic assessment on the functions of tenomodulin gene. Gene. 2016;587:1-17.

20. Docheva D, Hunziker EB, Fässler R, Brandau O. Tenomodulin is necessary for tenocyte proliferation and tendon maturation. Mol Cell Biol. 2005;25:699-705.

21. Oshima Y, Sato K, Tashiro F, Miyazaki J, Nishida K, Hiraki Y, et al. Anti-angiogenic action of the C-terminal domain of tenomodulin that shares homology with chondromodulin-I. J Cell Sci. 2004;117:2731-44.

22. Alberton P, Dex S, Popov C, Shukunami C, Schieker M, Docheva D. Loss of tenomodulin results in reduced self-renewal and augmented senescence of tendon stem/progenitor cells. Stem Cells Dev. 2015;24:597-609.

23. Yin H, Caceres MD, Yan Z, Schieker M, Nerlich M, Docheva D. Tenomodulin regulates matrix remodeling of mouse tendon stem/progenitor cells in an ex vivo collagen I gel model. Biochem Biophys Res Commun. 2019;512:691-7.

24. Dex S, Alberton P, Willkomm L, Söllradl T, Bago S, Milz S, et al. Tenomodulin is required for tendon endurance running and collagen I fibril adaptation to mechanical load. EBioMedicine. 2017;20:240-54.

25. Palmes D, Spiegel HU, Schneider TO, Langer M, Stratmann U, Budny T, et al. Achilles tendon healing: long-term biomechanical effects of postoperative mobilization and immobilization in a new mouse model. J Orthop Res. 2002;20:939-46.

26. Lin D, Alberton P, Caceres MD, Volkmer E, Schieker M, Docheva D. Tenomodulin is essential for prevention of adipocyte accumulation and fibrovascular scar formation during early tendon healing. Cell Death Dis. 2017;8:e3116.

27. Stoll C, John T, Conrad C, Lohan A, Hondke S, Ertel W, et al. Healing parameters in a rabbit partial tendon defect following tenocyte/biomaterial implantation. Biomaterials. 2011;32:4806-15.

28. Freedman BR, Sarver JJ, Buckley MR, Voleti PB, Soslowsky LJ. Biomechanical and structural response of healing Achilles tendon to fatigue loading following acute injury. J Biomech. 2014;47:2028-34.

29. Best TM, Collins A, Lilly EG, Seaber AV, Goldner R, Murrell GA. Achilles tendon healing: a correlation between functional and mechanical performance in the rat. J Orthop Res. 1993;11:897-906.

30. Dourte LM, Pathmanathan L, Jawad AF, lozzo RV, Mienaltowski MJ, Birk DE, et al. Influence of decorin on the mechanical, compositional, and structural properties of the mouse patellar tendon. J Biomech Eng. 2012;134:31005.

31. Hochstrat E, Müller M, Frank A, Michel P, Hansen U, Raschke MJ, et al. Cryopreservation of tendon tissue using dimethyl sulfoxide combines conserved cell vitality with maintained biomechanical features. PLoS ONE. 2019;14: e0215595. 
32. Picelli S, Faridani OR, Björklund AK, Winberg G, Sagasser S, Sandberg R. Full-length RNA-seq from single cells using Smart-seq2. Nat Protoc. 2014;9:171-81.

33. Shimono K, Uchibe K, Kuboki T, Iwamoto M. The pathophysiology of heterotopic ossification: Current treatment considerations in dentistry. Jpn Dent Sci Rev. 2014;50:1-8.

34. Kimura N, Shukunami C, Hakuno D, Yoshioka M, Miura S, Docheva D, et al. Local tenomodulin absence, angiogenesis, and matrix metalloproteinase activation are associated with the rupture of the chordae tendineae cordis. Circulation. 2008;118:1737-47.

35. Lin D, Alberton P, Delgado Caceres M, Prein C, Clausen-Schaumann H, Dong J, et al. Loss of tenomodulin expression is a risk factor for age-related intervertebral disc degeneration. Aging Cell. 2020;19:e13091.

36. Loiselle AE, Bragdon GA, Jacobson JA, Hasslund S, Cortes ZE, Schwarz EM, et al. Remodeling of murine intrasynovial tendon adhesions following injury: MMP and neotendon gene expression. J Orthop Res. 2009;27:833-40.

37. Taylor SH, Al-Youha S, van Agtmael T, Lu Y, Wong J, McGrouther DA, et al. Tendon is covered by a basement membrane epithelium that is required for cell retention and the prevention of adhesion formation. PLoS ONE. 2011;6:e16337.

38. Yoshida R, Alaee F, Dyrna F, Kronenberg MS, Maye P, Kalajzic I, et al. Murine supraspinatus tendon injury model to identify the cellular origins of rotator cuff healing. Connect Tissue Res. 2016;57:507-15.

39. Grcevic D, Pejda S, Matthews BG, Repic D, Wang L, Li H, et al. In vivo fate mapping identifies mesenchymal progenitor cells. Stem Cells. 2012;30:187-96.

40. Kalajzic Z, Li H, Wang L-P, Jiang X, Lamothe K, Adams DJ, et al. Use of an alphasmooth muscle actin GFP reporter to identify an osteoprogenitor population. Bone. 2008;43:501-10.

41. Moser HL, Doe AP, Meier K, Garnier S, Laudier D, Akiyama H, et al. Genetic lineage tracing of targeted cell populations during enthesis healing. J Orthop Res. 2018;36:3275-84.

42. Gracey E, Burssens A, Cambré I, Schett G, Lories R, Mclnnes IB, et al. Tendon and ligament mechanical loading in the pathogenesis of inflammatory arthritis. Nat Rev Rheumatol. 2020;16:193-207.

43. Asai S, Otsuru S, Candela ME, Cantley L, Uchibe K, Hofmann TJ, et al. Tendon progenitor cells in injured tendons have strong chondrogenic potential: the CD105-negative subpopulation induces chondrogenic degeneration. Stem Cells. 2014;32:3266-77.

44. Agarwal S, Loder SJ, Cholok D, Peterson J, Li J, Breuler C, et al. Scleraxis-lineage cells contribute to ectopic bone formation in muscle and tendon. Stem Cells. 2017;35:705-10.

45. Dey D, Bagarova J, Hatsell SJ, Armstrong KA, Huang L, Ermann J, et al. Two tissueresident progenitor lineages drive distinct phenotypes of heterotopic ossification. Sci Transl Med. 2016;8:366ra163.

46. Feng $H$, Xing W, Han $Y$, Sun J, Kong M, Gao B, et al. Tendon-derived cathepsin $\mathrm{K}$-expressing progenitor cells activate Hedgehog signaling to drive heterotopic ossification. J Clin Invest. 2020;130:6354-65.

47. Park NR, Shetye S, Keene DR, Tufa S, Hudson DM, Archer M, et al. Reticulocalbin 3 is involved in postnatal tendon development by regulating collagen fibrillogenesis and cellular maturation. Sci Rep. 2020;11:10868.

48. Ito S, Nagata K. Biology of Hsp47 (Serpin H1), a collagen-specific molecular chaperone. Semin Cell Dev Biol. 2017;62:142-51.

49. Stanley A, Heo S-J, Mauck RL, Mourkioti F, Shore EM. Elevated BMP and mechanical signaling through YAP1/RhoA poises FOP mesenchymal progenitors for osteogenesis. J Bone Min Res. 2019;34:1894-909.

50. Kishimoto $Y$, Ohkawara $B$, Sakai $T$, Ito $M$, Masuda $A$, Ishiguro $N$, et al. Wnt/ $\beta$-catenin signaling suppresses expressions of Scx, Mkx, and Tnmd in tendonderived cells. PLoS ONE. 2017;12:e0182051.

51. Miyabara S, Yuda Y, Kasashima Y, Kuwano A, Arai K. Regulation of tenomodulin expression via $W n t / \beta$-catenin signaling in equine bone marrow-derived mesenchymal stem cells. J Equine Sci. 2014;25:7-13.

\section{ACKNOWLEDGEMENTS}

MDC and DD acknowledge the German Research Foundation (DFG grant DO1414/3-1) and the EU Twinning Grant Achilles (H2020-WIDESPREAD-05-2017-Twinning Grant No. 810850) for the financial research support. DD thanks Dr. Ronen Schweitzer (Shriners Hospital for Children, Portland, USA) for providing ScxGFP reporter mice. We acknowledge Dr. Zexing Yan for excellent assisting during operations; Dr. Birgit Striegl (Regensburg Centre of Biomedical Engineering, Regensburg, Germany) for the support with $\mu C T$ measurements on DFG-funded instrument (Grant Nr. INST 102/11-1 FUGG); Dr. Sarah Hücker and Dr. Stefan Kirsch (Fraunhofer ITEM, Regensburg, Germany) for performing scRNA-Seq; and Heiko Siegmund (Institute for Pathology, Regensburg, Germany) for TEM assistance. We thank Dr. Girish Pattappa for English proof-reading.

\section{AUTHOR CONTRIBUTIONS}

MDC co-designed the study, allocated experimental groups, performed experiments, interpreted the results and wrote the manuscript; KA, MG and CGP contributed to operations; DL scored tissue sections; $\mathrm{XL}, \mathrm{ARV}$ and JW performed bioinformatics analysis; CB conducted TEM analysis; PM and RS supervised biomechanical analysis; VA approved the manuscript; DD planned and conceptualized the study, obtained third party funding, analyzed the data, interpreted the results and wrote the manuscript.

\section{FUNDING}

Open Access funding enabled and organized by Projekt DEAL.

\section{COMPETING INTERESTS}

The authors declare no competing interests.

\section{ADDITIONAL INFORMATION}

Supplementary information The online version contains supplementary material available at https://doi.org/10.1038/s41419-021-04298-z.

Correspondence and requests for materials should be addressed to Denitsa Docheva.

Reprints and permission information is available at http://www.nature.com/ reprints

Publisher's note Springer Nature remains neutral with regard to jurisdictional claims in published maps and institutional affiliations.

\begin{abstract}
Open Access This article is licensed under a Creative Commons adaptation, distribution and reproduction in any medium or format, as long as you give appropriate credit to the original author(s) and the source, provide a link to the Creative Commons license, and indicate if changes were made. The images or other third party material in this article are included in the article's Creative Commons license, unless indicated otherwise in a credit line to the material. If material is not included in the article's Creative Commons license and your intended use is not permitted by statutory regulation or exceeds the permitted use, you will need to obtain permission directly from the copyright holder. To view a copy of this license, visit http://creativecommons. org/licenses/by/4.0/.
\end{abstract}

(c) The Author(s) 2021 